\title{
On the Physical Realizability of a Class of Nonlinear Quantum Systems
}

\author{
Aline I. Maalouf and Ian R. Petersen \\ School of Engineering and Information Technology, \\ University of New South Wales at the Australian Defence Force Academy, \\ Canberra, ACT 2600 a.maalouf@adfa.edu.au i.r.petersen@gmail.com*
}

\begin{abstract}
In this paper, the physical realizability property is investigated for a class of nonlinear quantum systems. This property determines whether a given set of nonlinear quantum stochastic differential equations corresponds to a physical nonlinear quantum system satisfying the laws of quantum mechanics.
\end{abstract}

\section{Introduction}

In the physics literature, methods have been developed to model a wide range of open quantum systems, such as those encountered in quantum optics, within the framework of quantum stochastic differential equations [3], [9], [6]. In this context, quantum noise is used to represent the influence of large heat baths and boson fields, including optical and phonon fields, from which completely positive maps, Lindblad generators and master equations are obtained by taking expectations [10]. We may then distinguish two types of quantum stochastic differential equations, namely, linear and nonlinear. Linear quantum systems can be described by linear quantum stochastic differential equations and arise mostly in the area of quantum optics; e.g., see [7], [9] and [4]. An important class of linear quantum stochastic models describe the Heisenberg evolution of the annihilation and creation operators of several independent open quantum harmonic oscillators that are coupled to external coherent bosonic fields, such as coherent laser beams; e.g., see [8], [7] and [9]. A special class of these linear quantum systems is driven by quantum Wiener processes as in [2] where physical realizability conditions are developed to determine when the linear quantum system under consideration can be regarded as a representation of a linear quantum harmonic oscillator. This imposes some restrictions on the

\footnotetext{
${ }^{*}$ This work was completed with the support of a University of New South Wales Postgraduate Award and the Australian Research Council.
}

matrices describing the linear quantum model. This notion of physical realizability has been further investigated in [11] where the authors prove the equivalence between the algebraic conditions for physical realizability obtained in [2] and a frequency domain condition that an associated linear system is $(J, J)$-unitary. In fact, [11] extends the frequency domain physical realizability results of [12], [13] and [1] which apply to linear quantum systems described purely in terms of annihilation operators only to a more general class of linear quantum systems, which are described in terms of both the annihilation and creation operators. More explicitly, in [12], [13] and [1], the physical realizability conditions developed for annihilation-operator linear quantum systems have been related to the lossless property of linear systems and [11] generalizes this result to relate the corresponding physical realizability conditions of annihilation and creation operators linear quantum systems to the property of $(J, J)$-unitary. However, the question of addressing the physical realizability conditions for nonlinear quantum systems described by nonlinear quantum stochastic differential equations is still open. And in this paper, we restrict attention to a class of nonlinear quantum systems which is a generalization of the annihilation operator only linear quantum systems considered in [12], [13] and [1].

The paper is organized as follows: Section II defines the class of nonlinear quantum systems under consideration. Section III develops conditions under which the commutation relations for the nonlinear quantum systems are preserved. Section IV defines physical realizability in terms of nonlinear open quantum harmonic oscillators. Section V provides an example to illustrate the theory developed. Section VI concludes the paper.

\section{The Class of Nonlinear Quantum Sys- tems}

We consider an open quantum system $\mathrm{G}$ with physical variable space $\mathscr{A}_{G}$ consisting of operators defined 
on an underlying Hilbert space $\mathscr{H}_{G}$. The self-energy of this system is described by a Hamiltonian $\mathscr{H} \in \mathscr{A}_{G}$. The system is driven by $m$ field channels given by the quantum stochastic process $W$

$$
W=\left(\begin{array}{c}
W_{1} \\
\vdots \\
W_{m}
\end{array}\right) .
$$

These describe the annihilation of photons in the field channels and are operators on a Hilbert space $F$, with associated variable space $\mathscr{F}$. In that case, $F$ is the Hilbert space defining an indefinite number of quanta (called a Fock space [5]), and $\mathscr{F}$ is the space of operators over this space.

We assume the process $W$ is canonical, meaning that we have the following second order Ito products:

$$
\begin{aligned}
d W_{k}(t) d W_{l}(t)^{*} & =\delta_{k l} d t ; \\
d W_{k}(t)^{*} d W_{l}(t) & =0 ; \\
d W_{k}(t) d W_{l}(t) & =0 ; \\
d W_{k}(t)^{*} d W_{l}(t)^{*} & =0
\end{aligned}
$$

where $W_{k}(t)^{*}$ is the operator adjoint of $W_{k}(t)$ which is defined on the same Fock space.

The system is coupled to the field through a scattering matrix $S=I$ and a coupling vector of operators $L$ given by

$$
L=\left(\begin{array}{c}
L_{1} \\
\vdots \\
L_{m}
\end{array}\right)
$$

where $L_{j} \in \mathscr{A}_{G}$.

The notation $G=(S, L, H)$ is used to indicate an open quantum system specified by the parameters $S, L$ and $H$ where $H$ represents the Hamiltonian of the system. For $S=I$, the Schrodinger equation is given by

$$
d U(t)=\left\{d W^{\dagger} L-L^{\dagger} d W-\frac{1}{2} L^{\dagger} L d t-i H d t\right\} U(t)
$$

with the initial condition $U(0)=I$. Equation (1) determines the unitary motion of the system in accordance of the fundamental laws of quantum mechanics. Note that the notation ${ }^{\dagger}$ refers to the Hilbert space adjoint.

Given a system annihilation operator $a_{l} \in \mathscr{A}_{G}$, its Heisenberg evolution is defined by $a_{l}(t)=j_{t}\left(a_{l}\right)=$ $U(t)^{*} a_{l} U(t)$ and satisfies

$$
\begin{aligned}
d a_{l}(t)= & \left(\mathscr{L}_{L}(t)\left(a_{l}(t)\right)-i\left[a_{l}(t), H(t)\right]\right) \\
& +\left[L(t)^{\dagger}, a_{l}(t)\right] d W(t)
\end{aligned}
$$

where the notation $[A, B]=A B-B A$ denotes the commutator of two operators $A$ and $B$.
In equation (2), all operators evolve unitarily and the notation $\mathscr{L}_{L}\left(a_{l}\right)$ refers to

$$
\mathscr{L}_{L}\left(a_{l}\right)=\frac{1}{2} L^{\dagger}\left[a_{l}, L\right]+\frac{1}{2}\left[L^{\dagger}, a_{l}\right] L
$$

In this paper, we restrict attention to annihilation only coupling operators; i.e, the coupling operator $L(t)$ depends only on the annihilation operator $a_{l}(t)$ and not the creation operator $a_{l}(t)^{*}$. Therefore, $L(t)$ satisfies the following commutation property: $\left[a_{l}, L\right]=0$ and then

$$
\mathscr{L}_{L}\left(a_{l}\right)=\frac{1}{2}\left[L^{\dagger}, a_{l}\right] L
$$

The generator of the system $G$ is given by

$$
\mathscr{G}_{G}\left(a_{l}\right)=-i\left[a_{l}, H\right]+\mathscr{L}_{L}\left(a_{l}\right) .
$$

For the case of having $n$ annihilation operators $a_{1}, \ldots, a_{n}$, we define

$$
\begin{gathered}
a=\left(\begin{array}{c}
a_{1} \\
\vdots \\
a_{n}
\end{array}\right), \\
\mathscr{L}_{L_{i}}(a)=\frac{1}{2}\left[L^{\dagger}, a_{i}\right] L
\end{gathered}
$$

and

$$
\mathscr{G}_{G_{i}}(a)=-i\left[a_{i}, H\right]+\mathscr{L}_{L_{i}}(a) .
$$

Therefore, we can write

$$
\begin{aligned}
d a_{i}(t)= & \left(\mathscr{L}_{L_{i}}(t)\left(a_{i}(t)\right)-i\left[a_{i}(t), H(t)\right]\right) \\
& +\left[L(t)^{\dagger}, a_{i}(t)\right] d W(t)
\end{aligned}
$$

and

$$
\begin{aligned}
d a(t)= & \left(\mathscr{L}_{L}(t)(a(t))-i[a(t), H(t)]\right) \\
& +\left[L(t)^{\dagger}, a(t)\right] d W(t)
\end{aligned}
$$

where

$$
\mathscr{L}_{L}(a)=\left(\begin{array}{c}
\mathscr{L}_{L_{1}}(a) \\
\vdots \\
\mathscr{L}_{L_{n}}(a)
\end{array}\right)
$$

and

$$
[a, H]=\left(\begin{array}{c}
{\left[a_{1}, H\right]} \\
\vdots \\
{\left[a_{n}, H\right]}
\end{array}\right)
$$


Let

$$
\begin{aligned}
A\left(a(t), a(t)^{\dagger}\right) & =\left(\begin{array}{c}
A_{1}\left(a(t), a(t)^{\dagger}\right) \\
\vdots \\
A_{n}\left(a(t), a(t)^{\dagger}\right)
\end{array}\right) \\
& =\left(\begin{array}{c}
\mathscr{L}_{L_{1}}(a) \\
\vdots \\
\mathscr{L}_{L_{n}}(a)
\end{array}\right)-i\left(\begin{array}{c}
{\left[a_{1}, H\right]} \\
\vdots \\
{\left[a_{n}, H\right]}
\end{array}\right) \\
& =\mathscr{L}_{L}(a)-i[a, H] \\
& =\mathscr{G}_{G}(a)
\end{aligned}
$$

and

$$
\begin{aligned}
B\left(a(t), a(t)^{\dagger}\right) & =\left(\begin{array}{c}
B_{1}\left(a(t), a(t)^{\dagger}\right) \\
\vdots \\
B_{n}\left(a(t), a(t)^{\dagger}\right)
\end{array}\right) \\
& =\left(\begin{array}{c}
{\left[L^{\dagger}, a_{1}\right]} \\
\vdots \\
{\left[L^{\dagger}, a_{n}\right]}
\end{array}\right) \\
& =\left[L^{\dagger}, a\right] .
\end{aligned}
$$

Hence,

$$
\begin{aligned}
d a(t) & =A\left(a(t), a(t)^{\dagger}\right) d t+B\left(a(t), a(t)^{\dagger}\right) d W(t) \\
d a(t)^{*} & =A\left(a(t), a(t)^{\dagger}\right)^{*} d t+B\left(a(t), a(t)^{\dagger}\right)^{*} d W(t)^{*} .
\end{aligned}
$$

Note that for the case of matrices, the notations * and $\dagger$ refer respectively to the complex conjugate and the complex conjugate transpose of the matrix in question.

On the other hand, the components of the output fields are defined by $y(t)=j_{t}(W(t))=U(t)^{*} W(t) U(t)$ and satisfy the nonlinear quantum stochastic differential equations

$$
\begin{aligned}
d y(t) & =C(a(t)) d t+D(t) d W(t) \\
d y(t)^{*} & =C(a(t))^{*} d t+D(t)^{*} d W(t)^{*}
\end{aligned}
$$

where $C(a(t))=L(t), C(a(t))^{*}=L(t)^{*}, D(t)=I$ and $D(t)^{*}=I$. Hence, the system $G$ can be described by the following nonlinear quantum stochastic differential equations

$$
\begin{aligned}
d \bar{a}(t) & =\bar{A}\left(a(t), a(t)^{\dagger}\right) d t+\bar{B}\left(a(t), a(t)^{\dagger}\right) d \bar{W}(t) ; \\
d \bar{y}(t) & \left.=\bar{C}\left(a(t), a(t)^{\dagger}\right) d t+\bar{D}\left(a(t), a(t)^{\dagger}\right) d \bar{W}(\mathfrak{t}) 15\right)
\end{aligned}
$$

where $\bar{a}(t)=\left[\begin{array}{c}a \\ a^{*}\end{array}\right], \quad \bar{A}\left(a, a^{\dagger}\right)=\left[\begin{array}{c}A\left(a, a^{\dagger}\right) \\ A\left(a, a^{\dagger}\right)^{*}\end{array}\right]$,

$$
\bar{C}\left(a, a^{\dagger}\right)=\left[\begin{array}{c}
C(a) \\
C(a)^{*}
\end{array}\right], \quad \bar{B}\left(a, a^{\dagger}\right)=
$$

$\left[\begin{array}{cc}B\left(a, a^{\dagger}\right) & 0 \\ 0 & B\left(a, a^{\dagger}\right)^{*}\end{array}\right], \bar{D}(t)=\left[\begin{array}{cc}D(t) & 0 \\ 0 & D(t)^{*}\end{array}\right]$ and $d \bar{W}(t)=\left[\begin{array}{c}d W(t) \\ d W(t)^{*}\end{array}\right]$.

Definition 2.1 The class of nonlinear quantum system we consider in this paper are nonlinear quantum systems that can be represented by the QSDES (15) such that the matrices $A\left(a, a^{\dagger}\right)$ and $C(a)$ satisfy $\left[C(a), a^{T}\right]=$ $0,\left[A\left(a, a^{\dagger}\right), a^{T}\right]=-\left[a, A\left(a, a^{\dagger}\right)^{T}\right]$ and

$$
\begin{aligned}
A_{i}\left(a, a^{\dagger}\right) & =\sum_{k_{i}=0}^{m_{k_{i}}} \sum_{h_{i}=0}^{m_{h_{i}}} \sum_{l=1}^{n} \sum_{p=1}^{n} \alpha_{p l k_{i} h_{i}} a_{p}^{k_{i}}\left(a_{l}^{*}\right)^{h_{i}} \\
C_{v}(a) & =\sum_{k_{v}=0}^{m_{k_{v}}} \sum_{p=1}^{n} \beta_{k_{v} p} a_{p}^{k_{v}}
\end{aligned}
$$

for $i=1, \ldots, n$ and $v=1, \ldots, m$ with $m_{k_{i}}, m_{h_{i}}, m_{k_{v}}, \beta_{k_{v} p}$ and $\alpha_{\text {plk }} h_{i}$ integers.

Moreover, the matrices $A\left(a, a^{\dagger}\right), B\left(a, a^{\dagger}\right)$ and $C(a)$ satisfy the following property:

$$
\begin{gathered}
\frac{1}{2 \bar{n}}\left[\bar{A}\left(a, a^{\dagger}\right)^{\dagger} \bar{\theta}^{-1} \bar{a}, \bar{a}\right]-\frac{1}{2 \bar{n}}\left[\bar{a}^{\dagger} \bar{\theta}^{-1} \bar{A}\left(a, a^{\dagger}\right), \bar{a}\right]= \\
\bar{A}\left(a, a^{\dagger}\right)-\frac{1}{2} \bar{B}\left(a, a^{\dagger}\right) \bar{C}\left(a, a^{\dagger}\right)
\end{gathered}
$$

where $\bar{n}=\left.\sup _{1 \leq i \leq n}\left(k_{i}+h_{i}\right)\right|_{\alpha_{p l k_{i} h_{i} \neq 0}}, \bar{\Theta}=\left[\begin{array}{cc}\Theta & 0 \\ 0 & \Theta^{*}\end{array}\right]$ and $\Theta=\left[a, a^{\dagger}\right]$. For more information, on $\Theta$, refer to Section III below.

In the sequel, we assume that $d W(t)$ admits the following decomposition:

$$
d W(t)=\beta_{w}(t) d t+d \tilde{w}(t)
$$

where $\tilde{w}(t)$ is the noise part of $W(t)$ and $\beta_{w}(t)$ is an adapted process (see [5] and [6]). The noise $\tilde{w}(t)$ is a vector of quantum Weiner processes with Ito table

$$
d \tilde{w}(t) d \tilde{w}(t)^{\dagger}=F_{\tilde{w}} d t
$$

(see [5]) where $F_{\tilde{w}}$ is a non-negative definite Hermitian matrix. Here, the notation ${ }^{\dagger}$ represents the adjoint transpose of a vector of operators. Also, we assume the following commutation relations hold for the noise components:

$$
\left[d \tilde{w}(t), d \tilde{w}(t)^{\dagger}\right] \triangleq d \tilde{w}(t) d \tilde{w}(t)^{\dagger}-\left(d \tilde{w}(t)^{*} d \tilde{w}(t)^{T}\right)^{T}=T_{w} d t .
$$

Here $T_{w}$ is a Hermitian matrix and the notation ${ }^{T}$ denotes the transpose of a vector or matrix of operators. The noise processes can be represented as operators on an appropriate Fock space; for more details, see [5]. 
The process $\beta_{w}(t)$ represents variables of other systems which may be passed to the system (15) via an interaction. Therefore, it is required that $\beta_{w}(0)$ be an operator on a Hilbert space distinct from that of $a_{0}$ and the noise processes. We also assume that $\beta_{w}(t)$ commutes with $a(t)$ for all $t \geq 0$. Moreover, since $\beta_{w}(t)$ is an adapted process, we note that $\beta_{w}(t)$ also commutes with $d \tilde{w}(t)$ for all $t \geq 0$. We have also that $\left[a, d W^{\dagger}\right]=0$.

\section{Commutation Relations}

For the nonlinear quantum system (15), the initial system variables $a(0)=a_{0}$ consist of operators satisfying the commutation relations

$$
\begin{aligned}
& {\left[a_{j}(0), a_{k}^{*}(0)\right]=\Theta_{j k}, \quad j, k=1, \ldots, n .} \\
& {\left[\bar{a}_{j}(0), \bar{a}_{k}^{*}(0)\right]=\bar{\Theta}_{j k}, \quad j, k=1, \ldots, 2 n .}
\end{aligned}
$$

Here, the commutator is defined by $\left[a_{j}, a_{k}^{*}\right] \triangleq a_{j} a_{k}^{*}-$ $a_{k}^{*} a_{j}=\Theta_{j k}$ where $\Theta$ is a complex matrix with elements $\Theta_{j k}$. With $a^{T}=\left(a_{1}, \ldots, a_{n}\right)$, the relations (20) can be written as

$$
\begin{aligned}
& {\left[a, a^{\dagger}\right] } \triangleq a a^{\dagger}-\left(a^{*} a^{T}\right)^{T}=\Theta \\
& {\left[\bar{a}, \bar{a}^{\dagger}\right] \triangleq\left[\begin{array}{cc}
\Theta & 0 \\
0 & \Theta^{*}
\end{array}\right]=\bar{\Theta} . }
\end{aligned}
$$

\subsection{Preservation of the Commutation Rela- tions}

The following theorem provides an algebraic characterization of when the nonlinear quantum system (15) preserves the commutation relations (20) as time evolves.

Theorem 3.1 For the nonlinear quantum system (15), we have that $\left[\bar{a}_{p}(0), \bar{a}_{q}^{*}(0)\right]=\bar{\Theta}_{p q}$ implies $\left[\bar{a}_{p}(t), \bar{a}_{q}^{*}(t)\right]=\bar{\Theta}_{p q}$ for all $t \geq 0$ with $p, q=1 \ldots, 2 n$ if and only if

$$
\begin{aligned}
& {\left[\bar{A}\left(\bar{a}, \bar{a}^{\dagger}\right), \bar{a}^{\dagger}\right]+\left[\bar{a}, \bar{A}\left(\bar{a}, \bar{a}^{\dagger}\right)^{\dagger}\right]} \\
& \quad+\bar{B}\left(\bar{a}, \bar{a}^{\dagger}\right) T_{\bar{w}} \bar{B}\left(\bar{a}, \bar{a}^{\dagger}\right)^{\dagger}=0 ; \\
& {\left[\bar{B}\left(\bar{a}, \bar{a}^{\dagger}\right), \bar{a}^{\dagger}\right]=0 \quad \text { and }} \\
& {\left[\bar{a}, \bar{B}\left(\bar{a}, \bar{a}^{\dagger}\right)^{\dagger}\right]=0}
\end{aligned}
$$

with $T_{\bar{w}}=\left[\begin{array}{cc}T_{w} & 0 \\ 0 & T_{w}^{*}\end{array}\right]$.

\section{Physical Realizability And The Nonlin- ear Quantum Harmonic Oscillator}

In this section, we consider conditions under which a nonlinear quantum system of the form (15) corre- sponds to a nonlinear open quantum harmonic oscillator. Such a system is said to be physically realizable. The class of nonlinear open quantum harmonic oscillators under consideration are defined by the nonlinear coupling operator $\bar{L}$ and a nonlinear Hamiltonian $\bar{H}$. To derive a nonlinear system of the form (15) from a nonlinear open quantum harmonic oscillator defined by $\bar{L}$ and $\bar{H}$, we proceed as in Section II to get the following definition.

Definition 4.1 A nonlinear quantum system of the form (15) is said to be physically realizable if it is a representation of a nonlinear open quantum harmonic oscillator defined by a nonlinear coupling operator $\bar{L}$ and a nonlinear Hamiltonian $\bar{H}$, i.e., if there exist $\bar{H}$ and $\bar{L}$ such that the matrices $\bar{A}\left(a, a^{\dagger}\right), \bar{B}\left(a, a^{\dagger}\right), \bar{C}\left(a, a^{\dagger}\right)$ and $\bar{D}\left(a, a^{\dagger}\right)$ satisfy the following equations

$$
\begin{aligned}
\bar{A}\left(a, a^{\dagger}\right) & =\frac{1}{2}\left[\bar{L}^{\dagger}, \bar{a}\right] \bar{J}+i[\bar{H}, \bar{a}] \\
\bar{B}\left(a, a^{\dagger}\right) & =\left[\bar{L}^{\dagger}, \bar{a}\right] \bar{J} ; \\
\bar{C}\left(a, a^{\dagger}\right) & =\bar{L} ; \\
\bar{D}\left(a, a^{\dagger}\right) & =I ;
\end{aligned}
$$

where $\bar{J}=\left[\begin{array}{cc}I & 0 \\ 0 & -I\end{array}\right]$.

The following theorem provides necessary and sufficient conditions for a nonlinear quantum system of the form (15) to be physically realizable.

Theorem 4.1 A nonlinear quantum system of the form (15) is physically realizable with $T_{\bar{w}}=\bar{J}=\left[\begin{array}{cc}I & 0 \\ 0 & -I\end{array}\right]$ if and only if

$$
\begin{aligned}
& {\left[\bar{A}\left(a, a^{\dagger}\right), \bar{a}^{\dagger}\right]+\left[\bar{a}, \bar{A}\left(a, a^{\dagger}\right)^{\dagger}\right]} \\
& \quad+\bar{B}\left(a, a^{\dagger}\right) \bar{J} \bar{B}\left(a, a^{\dagger}\right)^{\dagger}=0 ; \\
& {\left[\bar{B}\left(a, a^{\dagger}\right), \bar{a}^{\dagger}\right]=0 ;} \\
& {\left[\bar{a}, \bar{B}\left(a, a^{\dagger}\right)^{\dagger}\right]=0 ;} \\
& \bar{B}\left(a, a^{\dagger}\right)=\left[\bar{C}\left(a, a^{\dagger}\right)^{\dagger}, \bar{a}\right] \bar{J} \quad \text { and } \\
& \bar{D}\left(a, a^{\dagger}\right)=I .
\end{aligned}
$$

In this case, the corresponding nonlinear self-adjoint Hamiltonian $\bar{H}$ is given by

$$
\bar{H}=\frac{i}{2 \bar{n}}\left(\bar{a}^{\dagger} \bar{\theta}^{-1} \bar{A}\left(a, a^{\dagger}\right)-\bar{A}\left(a, a^{\dagger}\right)^{\dagger} \bar{\theta}^{-1} \bar{a}\right)
$$

where $\bar{n}$ is as defined in Definition 2.1 and the corresponding coupling operator is $\bar{L}=\bar{C}\left(a, a^{\dagger}\right)$. 


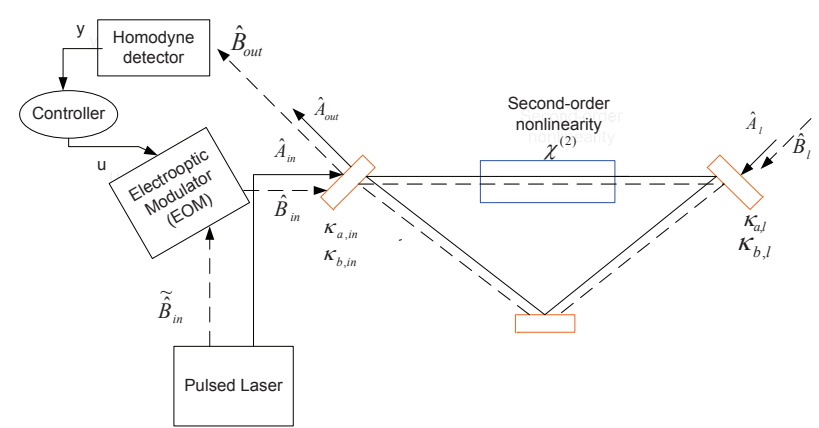

Figure 1. Schematic for the control of an optical parametric oscillator.

\section{Example}

We consider the application of the physical realizability conditions developed in this paper to an optical squeezer. A pulsed laser is used to drive the optical squeezer. The use of such a pulsed laser provides many advantages in an experimental situation; e.g, see [4].

The squeezer under consideration is implemented as an OPO driven by two time-varying optical fields; see Figure 1. A full description of the apparatus of Figure 1 is described in [14].

\subsection{Modeling}

The dynamics of the OPO system can be described as in [14] but with time-varying matrices and fields reflecting the fact that a pulsed laser is used to drive the experiment. The dynamics of the optical subsystem contain nonlinear terms. The quantities $A_{i n}$ and $B_{i n}$ are the input fields which set-up the fundamental and second-harmonic intracavity fields $a_{1}$ and $a_{2} . \kappa_{a_{i n}}$ and $\kappa_{b_{i n}}$ are the loss rates of the input/output mirrors for the $a_{1}$ and $a_{2}$ fields respectively. The parameters $\kappa_{a_{l}}$ and $\kappa_{b_{l}}$ are the internal loss rates for the corresponding two fields. Also, $\kappa_{a_{1}}=\kappa_{a_{i n}}+\kappa_{a_{l}}$ and $\kappa_{a_{2}}=\kappa_{b_{i n}}+\kappa_{b_{l}}$ are the associated total resonator decay rates. Furthermore, $\delta \hat{A}_{l}$ and $\delta \hat{B}_{l}$ represent the vacuum fields due to the internal losses. The output fields are given by $\hat{A}_{\text {out }}$ and $\hat{B}_{\text {out }}$.

The Heisenberg equations of motion are given by (see [14]):

$$
\begin{aligned}
& d a_{1}(t)=A_{1}\left(a, a^{\dagger}\right)(t) d t+B_{1}(t) d w_{1}(t) \\
& d a_{2}(t)=A_{2}\left(a, a^{\dagger}\right)(t) d t+B_{2}(t) d w_{2}(t) .
\end{aligned}
$$

In that case, $A_{1}\left(a, a^{\dagger}\right)=-\kappa_{a_{1}} a_{1}-2 \chi^{(2)} a_{1}^{*} a_{2}, B_{1}=$ $-\sqrt{2 \kappa_{a_{1}}}, A_{2}\left(a, a^{\dagger}\right)=-\kappa_{a_{2}} a_{2}+\chi^{(2)} a_{1}^{2}, B_{2}=-\sqrt{2 \kappa_{a_{2}}}$, $d w_{1}(t)=-d \hat{A}_{i n}$ and $d w_{2}(t)=-d \hat{B}_{i n} . a_{1}$ and $a_{2}$ satisfy the following commutation relations:

$$
\begin{aligned}
& {\left[a_{1}, a_{1}^{*}\right]=1 ;} \\
& {\left[a_{2}, a_{2}^{*}\right]=1 ;} \\
& {\left[a_{1}, a_{2}^{*}\right]=0 ;} \\
& {\left[a_{2}, a_{1}^{*}=0 ;\right.} \\
& {\left[a_{1}, a_{2}\right]=0 .}
\end{aligned}
$$

Let $a=\left[\begin{array}{l}a_{1} \\ a_{2}\end{array}\right], \quad A\left(a, a^{\dagger}\right)=\left[\begin{array}{l}A_{1}\left(a, a^{\dagger}\right) \\ A_{2}\left(a, a^{\dagger}\right)\end{array}\right], \quad B=$ $\left[\begin{array}{cc}B_{1} & 0 \\ 0 & B_{2}\end{array}\right], d w=\left[\begin{array}{l}d w_{1} \\ d w_{2}\end{array}\right], \bar{a}=\left[\begin{array}{c}a \\ a^{*}\end{array}\right], \bar{A}\left(a, a^{\dagger}\right)=$ $\left[\begin{array}{c}A\left(a, a^{\dagger}\right)^{*} \\ A\left(a, a^{\dagger}\right)^{*}\end{array}\right], \bar{B}\left[\begin{array}{cc}B & 0 \\ 0 & B^{*}\end{array}\right]$ and $d \bar{w}=\left[\begin{array}{c}d w \\ d w^{*}\end{array}\right]$.

On the other hand, we define

$$
d y(t)=C(a)(t) d t+D(t) d w(t)
$$

where $C(a)=\left[\begin{array}{c}\sqrt{2 \kappa_{a_{1}}} a_{1} \\ \sqrt{2 \kappa_{a_{2}}} a_{2}\end{array}\right]$ and $D(t)=\left[\begin{array}{cc}I & 0 \\ 0 & I\end{array}\right]$.

Also, we define $\bar{y}=\left[\begin{array}{c}y \\ y^{*}\end{array}\right], \bar{C}\left(a, a^{\dagger}\right)=\left[\begin{array}{c}C(a) \\ C(a)^{*}\end{array}\right]$, and $\bar{D}(t)=\left[\begin{array}{cc}D(t) & 0 \\ 0 & D(t)^{*}\end{array}\right]$.

Then, the OPO system can be described by

$$
\begin{aligned}
d \bar{a}(t) & =\bar{A}\left(a, a^{\dagger}\right)(t) d t+\bar{B}(t) d \bar{w}(t) ; \\
d \bar{y}(t) & =\bar{C}\left(a, a^{\dagger}\right)(t) d t+\bar{D}(t) d \bar{w}(t) .
\end{aligned}
$$

Note that this OPO system fits within the class of nonlinear quantum systems considered in this paper. In fact, the matrices $A\left(a, a^{\dagger}\right)$ and $C(a)$ satisfy $\left[C(a), a^{T}\right]=0$, $\left[A\left(a, a^{\dagger}\right), a^{T}\right]=-\left[a, A\left(a, a^{\dagger}\right)^{T}\right]$ and

$$
\begin{gathered}
\frac{1}{2 \bar{n}}\left[\bar{A}\left(a, a^{\dagger}\right)^{\dagger} \bar{\theta}^{-1} \bar{a}, \bar{a}\right]-\frac{1}{2 \bar{n}}\left[\bar{a}^{\dagger} \bar{\theta}^{-1} \bar{A}\left(a, a^{\dagger}\right), \bar{a}\right]= \\
\bar{A}\left(a, a^{\dagger}\right)-\frac{1}{2} \bar{B}\left(a, a^{\dagger}\right) \bar{C}\left(a, a^{\dagger}\right)
\end{gathered}
$$

where $\bar{n}=3, \bar{\Theta}=\left[\begin{array}{cc}I & 0 \\ 0 & -I\end{array}\right]$ and $\Theta=\left[a, a^{\dagger}\right]=I$.

In fact, $\frac{1}{2 \bar{n}}\left[\bar{A}\left(a, a^{\dagger}\right)^{\dagger} \bar{\theta}^{-1} \bar{a}, \bar{a}\right]=\left[\begin{array}{c}-\chi^{(2)} a_{1}^{*} a_{2} \\ \frac{1}{2} \chi^{(2)} a_{1}^{2} \\ -\chi^{(2)} a_{2}^{*} a_{1} \\ \frac{1}{2} \chi^{(2)} a_{1}^{2 *}\end{array}\right]$ and $\frac{1}{2 \bar{n}}\left[\bar{a}^{\dagger} \bar{\theta}^{-1} \bar{A}\left(a, a^{\dagger}\right), \bar{a}\right]=\left[\begin{array}{c}\chi^{(2)} a_{1}^{*} a_{2} \\ -\frac{1}{2} \chi^{(2)} a_{1}^{2} \\ \chi^{(2)} a_{2}^{*} a_{1} \\ -\frac{1}{2} \chi^{(2)} a_{1}^{2 *}\end{array}\right]$. On the other hand, $\bar{A}\left(a, a^{\dagger}\right)-\frac{1}{2} \bar{B}\left(a, a^{\dagger}\right) \bar{C}\left(a, a^{\dagger}\right)=$ 


$$
\begin{aligned}
& {\left[\begin{array}{c}
-2 \chi^{(2)} a_{1}^{*} a_{2} \\
\chi^{(2)} a_{1}^{2} \\
-2 \chi^{(2)} a_{2}^{*} a_{1} \\
\chi^{(2)} a_{1}^{2 *}
\end{array}\right] . \text { Hence, }} \\
& \frac{1}{2 \bar{n}}\left[\bar{A}\left(a, a^{\dagger}\right)^{\dagger} \bar{\theta}^{-1} \bar{a}, \bar{a}\right]-\frac{1}{2 \bar{n}}\left[\bar{a}^{\dagger} \bar{\theta}^{-1} \bar{A}\left(a, a^{\dagger}\right), \bar{a}\right]= \\
& \bar{A}\left(a, a^{\dagger}\right)-\frac{1}{2} \bar{B}\left(a, a^{\dagger}\right) \bar{C}\left(a, a^{\dagger}\right) .
\end{aligned}
$$

In addition, the system under consideration is physically realizable. In fact, $\bar{B}\left(a, a^{\dagger}\right)=\left[\bar{C}\left(a, a^{\dagger}\right)^{\dagger}, \bar{a}\right] \bar{J}=$

$\left[\begin{array}{cccc}-\sqrt{2 k_{a_{1}}} & 0 & 0 & 0 \\ 0 & -\sqrt{2 k_{a_{2}}} & 0 & 0 \\ 0 & 0 & -\sqrt{2 k_{a_{1}}} & 0 \\ 0 & 0 & 0 & -\sqrt{2 k_{a_{2}}}\end{array}\right]$. $\left[\bar{a}, \bar{B}\left(a, a^{\dagger}\right)^{\dagger}\right]=0$ and $\bar{D}\left(a, a^{\dagger}\right)=I$.

$$
\begin{gathered}
\text { Also, } \\
{\left[\begin{array}{cccc}
-k_{a_{1}} & -2 \chi^{(2)} a_{1}^{*} & 2 \chi^{(2)} a_{2} & 0 \\
2 \chi^{(2)} a_{1} & -k_{a_{2}} & 0 & 0 \\
-2 \chi^{(2)} a_{2}^{*} & 0 & k_{a_{1}} & 2 \chi^{(2)} a_{1} \\
0 & 0 & -2 \chi^{(2)} a_{1}^{*} & k_{a_{2}}
\end{array}\right],} \\
{\left[\bar{a}, \bar{A}\left(a, a^{\dagger}\right)^{\dagger}\right]=} \\
{\left[\begin{array}{cccc}
-k_{a_{1}} & 2 \chi^{(2)} a_{1}^{*} & -2 \chi^{(2)} a_{2} & 0 \\
-2 \chi^{(2)} a_{1} & -k_{a_{2}} & 0 & 0 \\
2 \chi^{(2)} a_{2}^{*} & 0 & k_{a_{1}} & -2 \chi^{(2)} a_{1} \\
0 & 0 & 2 \chi^{(2)} a_{1}^{*} & k_{a_{2}}
\end{array}\right]}
\end{gathered}
$$

and

$$
\bar{B}\left(a, a^{\dagger}\right) \bar{J} \bar{B}\left(a, a^{\dagger}\right)^{\dagger}
$$$$
\left[\begin{array}{cccc}
2 k_{a_{1}} & 0 & 0 & 0 \\
0 & 2 k_{a_{2}} & 0 & 0 \\
0 & 0 & -2 k_{a_{1}} & 0 \\
0 & 0 & 0 & -2 k_{a_{2}}
\end{array}\right] .
$$

Hence, $\quad\left[\bar{A}\left(a, a^{\dagger}\right), \bar{a}^{\dagger}\right]+\left[\bar{a}, \bar{A}\left(a, a^{\dagger}\right)^{\dagger}\right]+$ $\bar{B}\left(a, a^{\dagger}\right) \bar{J} \bar{B}\left(a, a^{\dagger}\right)^{\dagger}=0$.

In this case, the corresponding nonlinear selfadjoint Hamiltonian $\bar{H}$ is given by

$$
\begin{aligned}
\bar{H} & =\frac{i}{2 \bar{n}}\left(\bar{a}^{\dagger} \bar{\theta}^{-1} \bar{A}\left(a, a^{\dagger}\right)-\bar{A}\left(a, a^{\dagger}\right)^{\dagger} \bar{\theta}^{-1} \bar{a}\right) \\
& =i \chi^{(2)} a_{1}^{2 *} a_{2}-i \chi^{(2)} a_{2}^{*} a_{1}^{2} .
\end{aligned}
$$

and the corresponding coupling operator is $\bar{L}=$ $\bar{C}\left(a, a^{\dagger}\right)$.

\section{Conclusion}

In this paper, the issue of physical realizability for a class of nonlinear quantum systems is developed and verified by applying the theory developed to an optical squeezer. A future extension of this paper would be to relate the physical realizability properties to the lossless properties of the class of nonlinear quantum systems considered in this paper.

\section{References}

[1] A. I. Maalouf and I. R. Petersen, "Bounded real properties for a class of annihilation-operator linear quantum systems," IEEE Transactions on Automatic Control, vol. 56, no. 4, pp. 786 - 801, 2011.

[2] M. R. James, H. I. Nurdin, and I. R. Petersen, " $H^{\infty}$ control of linear quantum stochastic systems," IEEE Transactions on Automatic Control, vol. 53, no. 8, pp. 17871803, 2008.

[3] C. W. Gardiner and M. J. Collett, "Input and output in damped quantum systems: Quantum stochastic differential equations and the master equation," Physical Review $A$, vol. 31, no. 6, pp. 3761-3774, 1985.

[4] H. A. Bachor and T. C. Ralph, A Guide to Experiments in Quantum Optics. Weinheim, Germany:Wiley-VCH, 2004.

[5] K. R. Parthasarathy, An Introduction to Quantum Stochastic Calculus. Berlin: Birkhauser, 1992.

[6] R. L. Hudson and K. R. Parthasarathy, "Quantum Ito's formula and stochastic evolutions," Communications in Mathematical Physics, vol. 93, pp. 301-323, 1984.

[7] D. F. Walls and G. J. Milburn, Quantum optics. Berlin: Springer-Verlag, 2000.

[8] H. M. Wiseman and G. J. Milburn, Quantum Measurement and Control. Cambridge University Press: UK, 2010.

[9] C. W. Gardiner and P. Zoller, Quantum noise. Berlin: Springer, 2000.

[10] M. R. James and J. E. Gough, "Quantum Dissipative Systems and Feedback Control Design by Interconnection," IEEE Transactions on Automatic Control, vol. 55, no. 8, pp. 1806-1821, 2010.

[11] A. J. Shaiju and I. R. Petersen, "On the Physical Realizability of General Linear Quantum Stochastic Differential Equations with Complex Coefficients," Proceedings of the joint 48th IEEE Conference on Decision and Control and the 28th Chinese Control Conference, Shanghai, P.R. China, December 16-18, 2009, pp. 1422-1427, 2009.

[12] A. I. Maalouf and I. R. Petersen, "Coherent $\mathrm{H}^{\infty}$ Control for a Class of Linear Complex Quantum Systems," Proceedings of the American Control Conference ACC 2009, St. Louis, Missouri, 2009.

[13] A. I. Maalouf and I. R. Petersen, "Coherent $H^{\infty}$ Control for a class of annihilation-operator linear quantum systems," IEEE Transactions on Automatic Control, vol. 56, no. 2, pp. 309-319, 2011.

[14] S .Z .S . Hassen and I. R. Petersen and E. H. Huntington and M. Heurs and M. R. James, "LQG control of an optical squeezer," Proceedings of the American Control Conference ACC 2010, Baltimore, USA, pp.2730 - 2735, 2010. 